\title{
A third-order class-D amplifier with and without ripple compensation
}

\author{
Stephen M. Cox ${ }^{\mathrm{a}}$, H. du Toit Mouton ${ }^{\mathrm{b}}$ \\ ${ }^{a}$ School of Mathematical Sciences, University of Nottingham, University Park, Nottingham NGr 2RD, \\ United Kingdom \\ ${ }^{b}$ Department of Electrical and Electronic Engineering, Stellenbosch University, Private Bag X1, Matieland 7602, \\ South Africa
}

\begin{abstract}
We analyse the nonlinear behaviour of a third-order class-D amplifier, and demonstrate the remarkable effectiveness of the recently introduced ripple compensation (RC) technique in reducing the audio distortion of the device. The amplifier converts an input audio signal to a high-frequency train of rectangular pulses, whose widths are modulated according to the input signal (pulse-width modulation) and employs negative feedback. After determining the steady-state operating point for constant input and calculating its stability, we derive a small-signal model (SSM), which yields in closed form the transfer function relating (infinitesimal) input and output disturbances. This SSM shows how the RC technique is able to linearise the small-signal response of the device. We extend this SSM through a fully nonlinear perturbation calculation of the dynamics of the amplifier, based on the disparity in time scales between the pulse train and the audio signal. We obtain the nonlinear response of the amplifier to a general audio signal, avoiding the linearisation inherent in the SSM; we thereby more precisely quantify the reduction in distortion achieved through RC. Finally, simulations corroborate our theoretical predictions and illustrate the dramatic deterioration in performance that occurs when the amplifier is operated in an unstable regime. The perturbation calculation is rather general, and may be adapted to quantify the way in which other nonlinear negative-feedback pulse-modulated devices track a time-varying input signal that slowly modulates the system parameters.
\end{abstract}

Keywords: Class-D amplifier, pulse-modulated systems, PWM, piecewise-smooth systems

\footnotetext{
Email addresses: stephen.cox@nottingham.ac.uk (Stephen M. Cox), dtmouton@sun.ac.za (H. du Toit Mouton)
} 


\section{Introduction}

Class-D amplifiers are an important technological device and are widely used in mobile electronic devices, principally because of their exceptional efficiency [1], which helps improve battery life. They operate by converting an audio signal to a high-frequency train of rectangular pulses whose widths are modulated in a manner that depends on the audio signal (pulse-width modulation, PWM) [2]. They thus inherently involve dynamics on two different time scales and so are particularly amenable to analysis by perturbation methods.

To mitigate the influence of noise, designs for class-D amplifiers generally include some form of negative feedback, and hence may be modelled mathematically as piecewise-smooth dynamical systems [3, 4]. However, while theoretical interest in such systems has primarily focused on the existence, stability and bifurcations of various steady-state operating points (see, for example, [3, 4, 5, 6, 7]), here we are principally concerned with the regime of most practical interest, which is the nonlinear response to a relatively slowly varying audio input. Our goal is to understand the way in which the pulse-modulated system tracks a slowly varying input signal, with particular focus on the low-frequency components of the amplifier output.

In recent publications, we have explored the operation of relatively simple first- and secondorder designs of class-D amplifier (with, respectively, one or two integrators in the feedback path) [8, 9, 10, 11, 12] and we have illustrated the ripple compensation (RC) technique in the first-order case [10], However, in all these cases, the operation of the feedback loop is simple enough that the entire mathematical model may be reduced to one or two nonlinear scalar difference equations for the switching times of the output pulse-train. Here we treat a higher-order design, for which reduction to such a simple system is no longer possible, and the problem is formulated instead as a nonlinear system of difference equations with slowly varying forcing. Specifically, here we treat a more realistic mathematical model, with a third-order compensator in the feedback loop, and a second-order output filter, so that the state-space model for the amplifier is five-dimensional.

A key diagnostic of practical interest is the audio distortion that arises due to the nonlinearity of the switching in the negative feedback loop. Over the years, many techniques have been devised by engineers to reduce this inherent distortion, and thereby to improve the fidelity of the audio reproduction. The present paper is dedicated to examining the theoretical basis of the ripple compensation (RC) technique, which appears to be a particularly effective means of eliminating 
significant elements of the distortion [13, 14]. Calculation of the audio distortion is achieved by first considering the dynamics of the amplifier, specifically determining the relationship between the audio input and the switching times of the output pulse-train, then from these switching times determining the audio content of the output. Despite the algebraically involved nature of the problem, we are able to give explicit formulas for the principal components of the audio output of the amplifier; these expressions make clear the contribution of the $\mathrm{RC}$ technique towards linearising the output.

In Section 2, we describe the amplifier treated in this paper and the RC technique; we also formulate the state-space model for the device. In Section 3, we calculate the steady-state (timeperiodic) operating point of the device in response to a constant input, and briefly consider its stability in Section 4. This stability calculation informs the choice of parameter values for later simulations (our goal is not to explore instability and bifurcation, rather to ensure that the device is operated in a stable regime of practical relevance). In Section 5, we develop a small-signal model which yields a transfer function relating small disturbances at the input to the consequential small disturbances at the output. This small-signal model allows us to deduce certain aspects of the behaviour of the device in response to a full audio signal, and in particular shows the linearising effect of RC. The principal results of this paper are contained in Section 6, where we carry out a large-signal perturbation calculation of the amplifier output, where the perturbation parameter is proportional to the ratio between typical time scales for the output switching and the audio signal. We corroborate our theoretical results with corresponding simulations, which are presented in Section 7. Besides simulations in the stable regime of practical interest, we illustrate the calamitous sudden increase in the audio distortion that arises when the parameters of the device are poorly chosen, so that the steady-state operating point is unstable. Finally, we close, in Section 8, by summarising our results and emphasising that our analysis - in particular the asymptotic calculation of Section 6 - may be applied to a wide range of negative-feedback pulse-modulated systems. 




Figure 1: Third-order amplifier. The input audio signal is $u(t)$. $G$ represents the transfer function of the output filter. There is ripple compensation (RC) if $k=1$; otherwise, if $k=0$, there is no RC. The amplifier output is $g(t)$. The compensator is denoted by $H$. The output of the compensator, denoted by $m(t)$, is fed into the positive input of the comparator, whose negative input receives the sawtooth wave $v(t)$. The output takes the values \pm 1 according to the sign of $m(t)-v(t)$.

\section{Mathematical formulation}

Figure 1 shows the amplifier. The output $g(t)$ is a rectangular wave taking the values \pm 1 according to

$$
g(t)=\operatorname{sgn}(m(t)-v(t))
$$

where $m(t)$ and $v(t)$ are, respectively, the noninverting and inverting inputs of a comparator. The rising edges of $g(t)$ occur at regular intervals, where $t=n T$ and the constant $T$ is the period of the carrier wave $v(t)$. The falling edges of $g(t)$ occur at times that vary according to the output $m(t)$ of the compensator, as illustrated in Figure 2, We denote these modulated down-switching times by $A_{n}$, so that

$$
g(t)=\left\{\begin{aligned}
+1 & \text { for } n T<t<A_{n} \\
-1 & \text { for } A_{n}<t<(n+1) T
\end{aligned}\right.
$$

The sawtooth carrier wave is given by

$$
v(t)=-1+2(t-n T) / T
$$




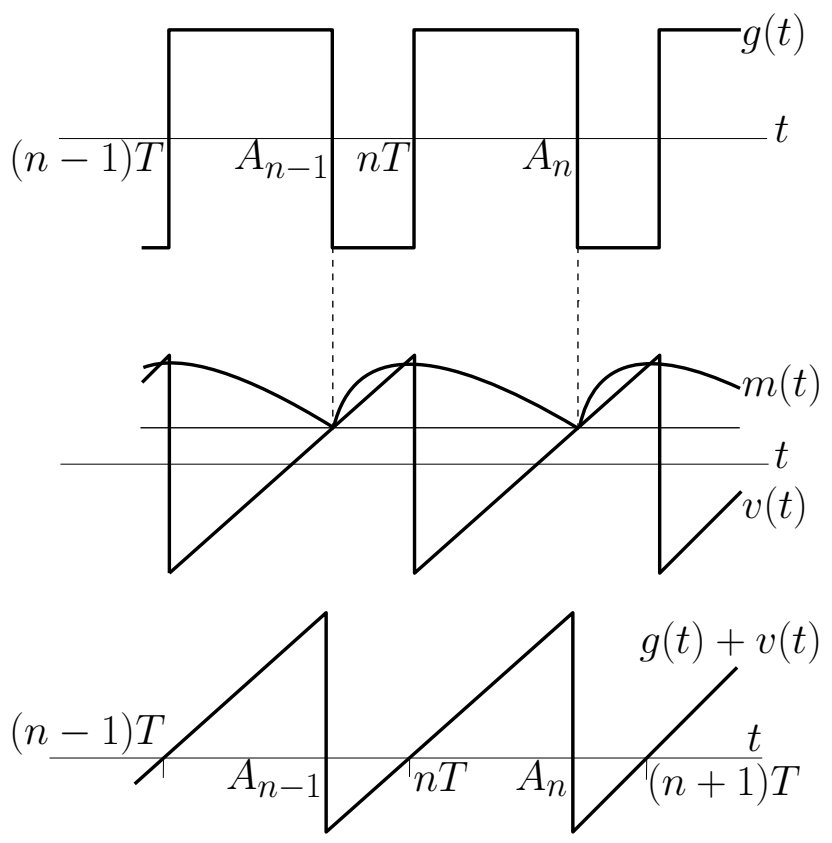

Figure 2: Signals $g(t), v(t), m(t)$ and $g(t)+v(t)$. The rising switching edges of $g(t)$ occur regularly, at times $t=n T$; the falling switching edges occur at times $t=A_{n}$, where $m\left(A_{n}\right)=v\left(A_{n}\right)$. (The signal $m(t)$ is for illustrative purposes.) The signal $g(t)+v(t)$ is a linear ramp, with falling switching edges at times $t=A_{n}$.

for $n T \leq t<(n+1) T$ (with $v(t+T)=v(t)$ for all $t$ ), and hence the condition for switching is

$$
m\left(A_{n}\right)=-1+2 a_{n}, \quad \text { where } a_{n}=\left(A_{n}-n T\right) / T .
$$

The low-pass filter $G$ receives as input $g(t)+k v(t)$, where $k$ is either 0 or 1 . The choice $k=0$ indicates that $\mathrm{RC}$ is not applied; we note that in this case the filter input is $g(t)$, which is piecewise constant, switches up at times $t=n T$ and down at times $t=A_{n}$. Otherwise, the choice $k=1$ corresponds to the application of RC; the filter input is now $g(t)+v(t)$, which is a piecewise linear upwards ramp, which switches down at times $t=A_{n}$, as in Figure 2. (Note that $G$ represents the output filter; its repositioning into the feedback loop, as in Figure 1. may be achieved through standard block-diagram manipulations - for example, see Figure 5 of [13].)

The motivation for the $\mathrm{RC}$ technique is the observation that feedback of the output ripple signal leads to a nonlinearity in the PWM process: high-frequency carrier components are aliased to the audio band, leading to distortion in the output signal of the amplifier [10, 15]. The analysis of [15] identifies mechanisms for distortion in both amplitude and phase. This distortion can be largely mitigated by cancelling the unmodulated switching edges at $t=n T$ [14]. Details are described in 


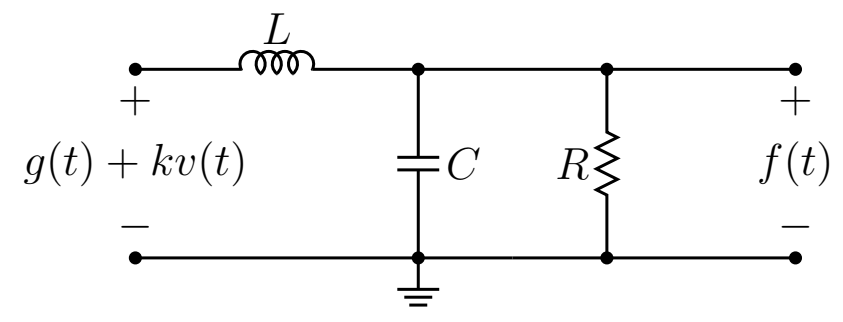

Figure 3: The second-order filter, $G$.

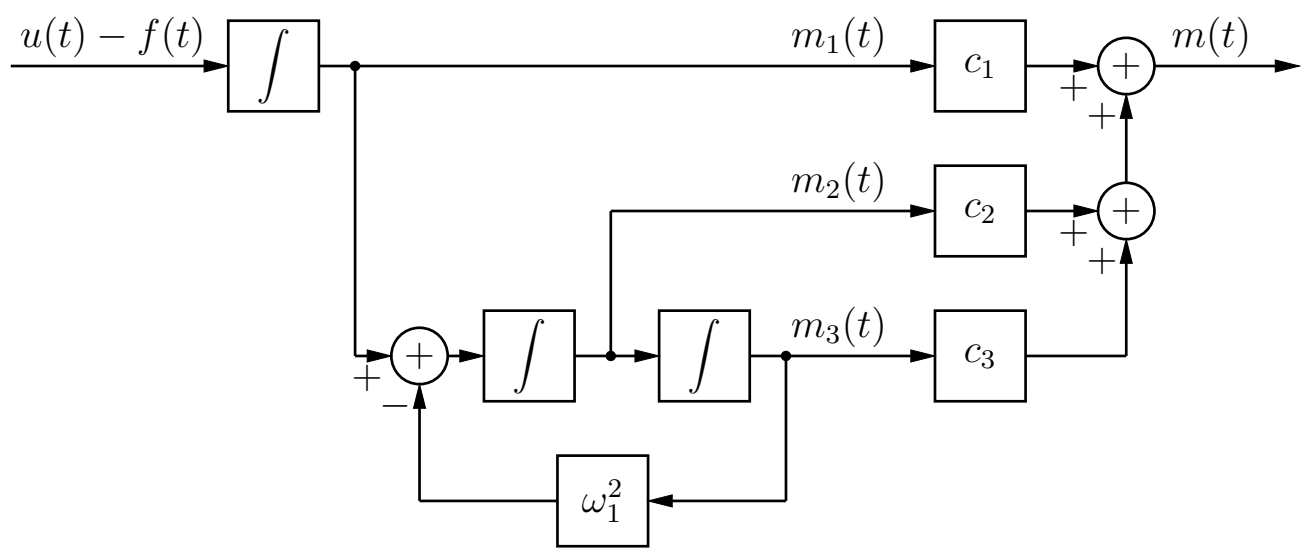

Figure 4: The third-order compensator, $H$.

Section 3 of [13], but the key to the success of RC in reducing output distortion may be illustrated 
a vector or matrix, and the prime denotes the time derivative. Then

$$
\boldsymbol{f}^{\prime}(t)=M_{2} \boldsymbol{f}(t)+\frac{g(t)+k v(t)}{L C}(0,1)^{T},
$$

where

$$
M_{2}=\left(\begin{array}{cc}
0 & 1 \\
-(L C)^{-1} & -(R C)^{-1}
\end{array}\right) .
$$

The filter may equivalently be specified in terms of its (Laplace) transfer function

$$
G(s)=1 /\left(L C s^{2}+L s / R+1\right) .
$$

Next we turn to the compensator $H$, which comprises a chain of integrators with feed-forward summation and a local resonator feedback loop, shown in Figure 4. We introduce the state vector $\boldsymbol{m}(t)=\left(m_{1}(t), m_{2}(t), m_{3}(t)\right)^{T}$. Then

$$
\boldsymbol{m}^{\prime}(t)=M_{3} \boldsymbol{m}(t)+(u(t)-f(t))(1,0,0)^{T},
$$

where

$$
M_{3}=\left(\begin{array}{ccc}
0 & 0 & 0 \\
1 & 0 & -\omega_{1}^{2} \\
0 & 1 & 0
\end{array}\right) .
$$

The output of the compensator is

$$
m(t)=c_{1} m_{1}(t)+c_{2} m_{2}(t)+c_{3} m_{3}(t)
$$

for some constants $c_{1}, c_{2}, c_{3}$. The (Laplace) transfer function of the compensator is

$$
H(s)=\frac{c_{1}}{s T}+\frac{c_{2}}{\left(\omega_{1}^{2}+s^{2}\right) T^{2}}+\frac{c_{3}}{\left(\omega_{1}^{2}+s^{2}\right) s T^{3}} .
$$

We solve the systems (3) and (4) together, by introducing the state-space vector

$$
\boldsymbol{x}(t)=\left(m_{1}(t), m_{2}(t), m_{3}(t), f(t), f^{\prime}(t)\right)^{T},
$$

which is governed by

$$
\boldsymbol{x}^{\prime}(t)=N \boldsymbol{x}(t)+u(t) \boldsymbol{e}_{1}+\frac{g(t)+k v(t)}{L C} \boldsymbol{e}_{5},
$$


where $\boldsymbol{e}_{1}=(1,0,0,0,0)^{T}, \ldots, \boldsymbol{e}_{5}=(0,0,0,0,1)^{T}$. The matrix $N$ is partitioned as follows:

$$
N=\left(\begin{array}{ccccc} 
& & & -1 & 0 \\
& M_{3} & & 0 & 0 \\
& & & 0 & 0 \\
0 & 0 & 0 & & \\
0 & 0 & 0 & &
\end{array}\right) .
$$

To simplify the analysis here and in later sections, we next diagonalise $N$. We thus introduce the diagonal matrix $\Lambda$ of the eigenvalues of $N$, which are $0, i \omega_{1},-i \omega_{1},-\mu+i \Omega$ and $-\mu-i \Omega$, where

$$
\mu=\frac{1}{2 R C}, \quad \Omega=\sqrt{\frac{1}{L C}-\frac{1}{4 R^{2} C^{2}}} .
$$

We also introduce a matrix $R$ whose columns are given, respectively, by the corresponding right eigenvectors of $N: \boldsymbol{w}_{1}, \ldots, \boldsymbol{w}_{5}$. Then

$$
N=R \Lambda R^{-1}
$$

The rows of $R^{-1}$ are the left eigenvectors of $N: \boldsymbol{v}_{1}, \ldots, \boldsymbol{v}_{5}$. Of particular utility in our analysis will be the left zero eigenvector

$$
\boldsymbol{v}_{1}=\left(-(L C)^{-1}, 0,0,(R C)^{-1}, 1\right)
$$

Correspondingly, $\boldsymbol{w}_{1}=\left(-L C, 0,-L C / \omega_{1}^{2}, 0,0\right)^{T}$.

To simplify later notation when we integrate (5), we introduce $\boldsymbol{P}_{n}(t)$ and $\boldsymbol{Q}_{n}(t)$, where

$$
\boldsymbol{P}_{0}(t)=\mathrm{e}^{N t} \boldsymbol{e}_{1}, \quad \text { and } \quad \boldsymbol{P}_{n+1}(t)=\int_{0}^{t} \boldsymbol{P}_{n}(\tau) \mathrm{d} \tau
$$

for $n=0,1, \ldots$;

$$
\boldsymbol{Q}_{0}(t)=\mathrm{e}^{N t} \boldsymbol{e}_{5}, \quad \text { and } \quad \boldsymbol{Q}_{n+1}(t)=\int_{0}^{t} \boldsymbol{Q}_{n}(\tau) \mathrm{d} \tau
$$

for $n=0,1, \ldots$. We find

$$
\boldsymbol{P}_{n}(t)=\frac{t^{n}}{n !} \boldsymbol{e}_{1}+\phi_{n}(t) \boldsymbol{e}_{2}+\phi_{n+1}(t) \boldsymbol{e}_{3}
$$

where $\phi_{-1}(t)=\cos \omega_{1} t$ and

$$
\phi_{n+1}(t)=\int_{0}^{t} \phi_{n}(\tau) \mathrm{d} \tau \quad \text { for } n=-1,0,1, \ldots
$$


We may readily integrate (5) over any time interval $\left[t_{0}, t_{1}\right]$, to give

$$
\boldsymbol{x}\left(t_{1}\right)=\mathrm{e}^{N\left(t_{1}-t_{0}\right)} \boldsymbol{x}\left(t_{0}\right)+\int_{t_{0}}^{t_{1}} \mathrm{e}^{N\left(t_{1}-\tau\right)} \boldsymbol{e}_{1} u(\tau) \mathrm{d} \tau+\frac{1}{L C} \int_{t_{0}}^{t_{1}} \mathrm{e}^{N\left(t_{1}-\tau\right)} \boldsymbol{e}_{5}(g(\tau)+k v(\tau)) \mathrm{d} \tau .
$$

For later purposes, our specific interest is in integrating (5) over the interval $\left[A_{n}, A_{n+1}\right]$ between successive falling edges of the amplifier output $g(t)$. To evaluate the first integral in (9), we begin by writing

$$
\mathcal{I}_{u, n} \equiv \int_{A_{n}}^{A_{n+1}} \mathrm{e}^{N\left(A_{n+1}-\tau\right)} \boldsymbol{e}_{1} u(\tau) \mathrm{d} \tau .
$$

Then Taylor expansion of $u(\tau)$ about $\tau=A_{n}$ and repeated use of integration by parts on the result, together with (8), gives

$$
\mathcal{I}_{u, n}=\sum_{k=0}^{\infty} \boldsymbol{P}_{k+1}\left(A_{n+1}-A_{n}\right) u^{(k)}\left(A_{n}\right),
$$

where the superscript denotes the $k$-th derivative. The second integral in (9) is similarly found (after just one integration by parts, to deal with $v(\tau)$ ) to be

$$
\begin{aligned}
\mathcal{I}_{g, v, n} & \equiv \int_{A_{n}}^{A_{n+1}} \mathrm{e}^{N\left(A_{n+1}-\tau\right)} \boldsymbol{e}_{5}(g(\tau)+k v(\tau)) \mathrm{d} \tau \\
& =2(1-k) \boldsymbol{Q}_{1}\left(a_{n+1} T\right)+\left(-1-k+2 k a_{n}\right) \boldsymbol{Q}_{1}\left(A_{n+1}-A_{n}\right)+\frac{2 k}{T} \boldsymbol{Q}_{2}\left(A_{n+1}-A_{n}\right) .
\end{aligned}
$$

Assembling these results, we thus arrive at the discrete-time model

$$
\boldsymbol{x}\left(A_{n+1}\right)=\mathrm{e}^{N\left(A_{n+1}-A_{n}\right)} \boldsymbol{x}\left(A_{n}\right)+\mathcal{I}_{u, n}+\frac{1}{L C} \mathcal{I}_{g, v, n},
$$

together with the switching condition (2), which becomes

$$
\boldsymbol{\gamma}^{T} \boldsymbol{x}\left(A_{n+1}\right)=-1+2 a_{n+1},
$$

where

$$
\gamma^{T}=\left(c_{1}, c_{2}, c_{3}, 0,0\right)
$$

The system (11), (12) forms the basis for our mathematical analysis of the amplifier. We note that this system may be reduced to a single (fifth-order, nonlinear) scalar difference equation for $a_{n}$ (cf. [16]). However, while we shall make use of a related reduction later, in Section [6, the bulk of our analysis concerns the formulation in (11), (12). 


\section{Steady-state operation}

We begin our analysis of the mathematical model set out above by examining its steady-state behaviour in response to a constant input. This is necessary in order for us to choose suitable parameter values for our simulations, where the steady-state response should be stable; it also sheds some light on the operation of RC.

We thus suppose that $u(t)=u_{0}$ and that all signals are $T$-periodic. In particular all duty cycles are equal, with $a_{n} \equiv a$. In such steady-state operation, (11) becomes

$$
\left(I_{5}-\mathrm{e}^{N T}\right) \boldsymbol{x}(a T)=\boldsymbol{\Phi}(a, T)
$$

where $I_{5}$ is the $5 \times 5$ identity matrix,

$$
\boldsymbol{\Phi}(a, T)=u_{0} \boldsymbol{P}_{1}(T)+\frac{1}{L C}\left\{2(1-k) \boldsymbol{Q}_{1}(a T)+(-1-k+2 k a) \boldsymbol{Q}_{1}(T)+\frac{2 k}{T} \boldsymbol{Q}_{2}(T)\right\},
$$

and the switching condition is $\boldsymbol{\gamma}^{T} \boldsymbol{x}(a T)=-1+2 a$. Note that the matrix on the left-hand side of (13) is singular, and after left-multiplying by $\boldsymbol{v}_{1}$, given in (7), we obtain the solvability constraint

$$
\boldsymbol{v}_{1} \boldsymbol{\Phi}(a, T)=0
$$

this then yields the duty-cycle condition

$$
a=\frac{1}{2}\left(1+u_{0}\right)
$$

which expresses the fact that the time-averaged output $\langle g(t)\rangle=u_{0}$.

Once the duty-cycle condition (14) has been imposed, it remains to determine $\boldsymbol{x}(a T)$, from which the entire periodic solution may subsequently be obtained using (9). This is accomplished by replacing one row (for example, the first) of the vector equation (13) with the switching condition (12); thus we solve

$$
\tilde{M} \boldsymbol{x}(a T)=\tilde{\boldsymbol{\Phi}}
$$

where

$$
\tilde{M}_{i j}= \begin{cases}c_{j} & \text { for } i=1 \text { and } j=1,2,3, \\ 0 & \text { for } i=1 \text { and } j=4,5, \\ \left(I_{5}-\mathrm{e}^{N T}\right)_{i j} & \text { for } i=2,3,4,5\end{cases}
$$

and

$$
\tilde{\Phi}_{i}= \begin{cases}-1+2 a & \text { for } i=1 \\ \Phi_{i}(a, T) & \text { for } i=2,3,4,5\end{cases}
$$


Explicit formulas for the steady-state solution are too algebraically involved to record here.

A quantity of particular significance in our later stability calculation and in our development

150

and

$$
\begin{aligned}
& \boldsymbol{x}_{b}^{\prime}(t)=N \boldsymbol{x}_{b}(t)+(2 b-1) \boldsymbol{e}_{1}+\frac{2}{L C}(-1+t / T) \boldsymbol{e}_{5} \quad \text { for } b T \leq t<b T+T, \\
& \boldsymbol{\gamma}^{T} \boldsymbol{x}_{b}(b T)=-1+2 b .
\end{aligned}
$$

To demonstrate the relationship between the two solutions, we introduce the $T$-periodic quantity

$$
\boldsymbol{\Delta}(t)=\boldsymbol{x}_{b}(t+(b-a) T)-\boldsymbol{x}_{a}(t),
$$

which satisfies

$$
\begin{aligned}
& \boldsymbol{\Delta}^{\prime}(t)=N \boldsymbol{\Delta}(t)+2(b-a)\left(e_{1}+\frac{1}{L C} e_{5}\right) \quad \text { for } a T \leq t<a T+T, \\
& \boldsymbol{\gamma}^{T} \boldsymbol{\Delta}(a T)=2(b-a) .
\end{aligned}
$$

The general solution to the ODE in (19) is

$$
\boldsymbol{\Delta}(t)=\mathrm{e}^{N(t-a T)} \boldsymbol{\Delta}(a T)+2(b-a) \int_{a T}^{t} \mathrm{e}^{N(t-\tau)} \boldsymbol{\epsilon} \mathrm{d} \tau,
$$

where

$$
\boldsymbol{\epsilon}=\boldsymbol{e}_{1}+\frac{1}{L C} \boldsymbol{e}_{5}
$$


From (20), we see that

$$
N \boldsymbol{\Delta}(t)=\mathrm{e}^{N(t-a T)} N \boldsymbol{\Delta}(a T)+2(b-a)\left(\mathrm{e}^{N(t-a T)}-I_{5}\right) \boldsymbol{\epsilon},
$$

which may be rearranged as

$$
\boldsymbol{X}(t)=\mathrm{e}^{N(t-a T)} \boldsymbol{X}(a T),
$$

where

$$
\boldsymbol{X}(t)=N \boldsymbol{\Delta}(t)+2(b-a) \boldsymbol{\epsilon} .
$$

Since $\boldsymbol{X}(t)$ is $T$-periodic, it follows from (21) with $t=a T+T$ that

$$
\left(\mathrm{e}^{N T}-I_{5}\right) \boldsymbol{X}(a T)=\mathbf{0} .
$$

Hence $\boldsymbol{X}(a T)=X_{3} \boldsymbol{w}_{1}$ for some constant $X_{3}$. The value of $X_{3}$ may be determined from (22): we see that

$$
\boldsymbol{v}_{1} \boldsymbol{X}(a T)=\boldsymbol{v}_{1} N \boldsymbol{\Delta}(a T)+2(b-a) \boldsymbol{v}_{1} \boldsymbol{\epsilon}=0
$$

hence (considering leftmost and rightmost sides of this equation) $X_{3}=0$. Thus $\boldsymbol{X}(a T)=\mathbf{0}$, and from (21) it follows that $\boldsymbol{X}(t) \equiv \mathbf{0}$, so that, from (22),

$$
\boldsymbol{\Delta}(t)=\left(\omega_{1}^{2}, 0,1,0,0\right)^{T} \Delta_{3}(t)+(0,0,0,2(b-a), 0)^{T},
$$

for some $\Delta_{3}(t)$. Substitution in (19) shows that $\boldsymbol{\Delta}(t)$ is in fact constant. Then from the switching condition in (19) we see that $\left(c_{1} \omega_{1}^{2}+c_{3}\right) \Delta_{3}=2(b-a)$, hence

$$
\boldsymbol{\Delta}=\frac{2(b-a)}{c_{1} \omega_{1}^{2}+c_{3}}\left(\omega_{1}^{2}, 0,1, c_{1} \omega_{1}^{2}+c_{3}, 0\right)^{T} .
$$

In summary, we have shown that, for $a T \leq t<a T+T$,

$$
\boldsymbol{x}_{b}(t+(b-a) T)=\boldsymbol{x}_{a}(t)+\boldsymbol{\Delta} .
$$

Since the two steady-state solutions differ by the addition of a constant vector, and by timeshifting, the derivatives of each solution around the modulated switching instant agree: more specifically,

$$
\boldsymbol{\gamma}^{T} \boldsymbol{x}_{b}^{\prime}(b T)=\gamma^{T} \boldsymbol{x}_{a}^{\prime}(a T)
$$

This fact has significant consequences, as we shall see later, in Section 5, when we show how it leads to a linearisation of the small-signal model. 


\section{Stability of the steady-state operating point}

Our interest is in stable operation of the amplifier, so we provide just a brief discussion of stability considerations. Following Aizerman and Gantmakher [17] (see also, for example, [3, 7, 18]), we suppose that the input $u(t)=u_{0}$ is fixed, and consider the growth or decay of a perturbation to the steady state over the interval $t \in[0, T]$. We write

$$
\boldsymbol{x}(t)=\overline{\boldsymbol{x}}(t)+\Delta \boldsymbol{x}(t), \quad a_{0}=a+\Delta a,
$$

where $\overline{\boldsymbol{x}}(t)$ is the steady-state solution with duty cycle $a$. Then, upon linearising in small disturbances, we find

$$
\Delta \boldsymbol{x}(T)=\underbrace{\mathrm{e}^{N(1-a) T}\left(I_{5}+\frac{T \kappa}{L C} \boldsymbol{e}_{5} \boldsymbol{\gamma}^{T}\right) \mathrm{e}^{N a T}}_{\equiv \mathcal{M}} \Delta \boldsymbol{x}(0)
$$

where

$$
\kappa=\left(1-\frac{1}{2} T \boldsymbol{\gamma}^{T} \overline{\boldsymbol{x}}^{\prime}(a T)\right)^{-1}
$$

and the quantity $\boldsymbol{\gamma}^{T} \overline{\boldsymbol{x}}^{\prime}(a T)$ may be obtained from (16). The stability of the steady-state operating point is thus determined by the eigenvalues of the matrix $\mathcal{M}[3]$. Note that the sole difference between the $\mathrm{RC}$ and no- $\mathrm{RC}$ versions of $\mathcal{M}$ lies in the value of $\kappa$.

The eigenvalues $\mu$ of $\mathcal{M}$ satisfy

$$
\operatorname{det}(\mathcal{M}-\mu I)=0
$$

We may derive an alternative equation for these eigenvalues (cf. [7, 19, 20]), which may be more useful in some cases, by use of Sylvester's Determinant Theorem [21], which states that $\operatorname{det}\left(I_{n}-\right.$ $A B)=\operatorname{det}\left(I_{p}-B A\right)$, where $A$ is any $n \times p$ matrix and $B$ is any $p \times n$ matrix, and $I_{n}$ and $I_{p}$ are, respectively, $n \times n$ and $p \times p$ identity matrices. We suppose, as is readily verified, that none of the eigenvalues of $\exp (N T)$ are also eigenvalues of $\mathcal{M}$. Then

$$
\operatorname{det}(\mathcal{M}-\mu I)=\operatorname{det}\left(\mathrm{e}^{N T}-\mu I+\boldsymbol{\alpha} \boldsymbol{\beta}^{T}\right)=\operatorname{det}\left(\mathrm{e}^{N T}-\mu I\right) \operatorname{det}\left(I+\left(\mathrm{e}^{N T}-\mu I\right)^{-1} \boldsymbol{\alpha} \boldsymbol{\beta}^{T}\right)
$$

where

$$
\boldsymbol{\alpha}=\frac{T \kappa}{L C} \mathrm{e}^{N(1-a) T} \boldsymbol{e}_{5}, \quad \boldsymbol{\beta}^{T}=\boldsymbol{\gamma}^{T} \mathrm{e}^{N a T} .
$$

Hence the eigenvalues $\mu$ of $\mathcal{M}$ satisfy

$$
\operatorname{det}\left(I+\left(\mathrm{e}^{N T}-\mu I\right)^{-1} \boldsymbol{\alpha} \boldsymbol{\beta}^{T}\right)=0
$$


The linearised switching condition (12) yields

$$
\Delta a_{n}=\frac{1}{2} \kappa \gamma^{T} \Delta \boldsymbol{x}\left(\bar{A}_{n}\right),
$$


where again $\kappa$ is given by (25). We note that when $k=0, \kappa$ depends on $u_{0}$, whereas when $k=1$, $\kappa$ is independent of $u_{0}$.

Through repeated integration by parts, as in the derivation of (10), it may be established, using (29) and (30), that

$$
\Delta \boldsymbol{x}\left(\bar{A}_{n+1}\right)=\mathcal{N} \Delta \boldsymbol{x}\left(\bar{A}_{n}\right)+\sum_{m=0}^{\infty} \boldsymbol{P}_{m+1}(T) \Delta u^{(m)}\left(\bar{A}_{n}\right)
$$

where

$$
\mathcal{N}=\mathrm{e}^{N T}\left(I_{5}+\frac{T \kappa}{L C} e_{5} \gamma^{T}\right)
$$

(Note that $\mathcal{M}=\mathrm{e}^{-N a T} \mathcal{N} \mathrm{e}^{N a T}$, where $\mathcal{M}$ is defined in (24), hence $\mathcal{M}$ and $\mathcal{N}$ are similar matrices and so share the same eigenvalues.)

A recurrence relation for the switching-time perturbation may now be derived by premultiplying (31) by $\frac{1}{2} \kappa \gamma^{T}$ then using (30). A convenient formulation for the solution may be obtained by introducing the derivative operator $\mathrm{D} \equiv \mathrm{d} / \mathrm{d} t$ and using the Taylor expansion [22, 23]

$$
\Delta \boldsymbol{x}\left(\bar{A}_{n+1}\right)=\mathrm{e}^{T \mathrm{D}} \Delta \boldsymbol{x}\left(\bar{A}_{n}\right)
$$

to give the formal solution

$$
\Delta a_{n}=\frac{1}{2} \kappa \gamma^{T}\left(\mathrm{e}^{T \mathrm{D}} I_{5}-\mathcal{N}\right)^{-1} \sum_{m=0}^{\infty} \boldsymbol{P}_{m+1}(T) \Delta u^{(m)}\left(\bar{A}_{n}\right)
$$

The next step is to characterise the corresponding spectral components of the output pulsetrain. To this end, we let $x(t)$ be such that $x\left(\bar{A}_{n}\right)=\Delta a_{n}$. In view of (32), one particular choice of $x(t)$ satisfies

$$
x(t)=\frac{1}{2} \kappa \boldsymbol{\gamma}^{T}\left(\mathrm{e}^{T \mathrm{D}} I_{5}-\mathcal{N}\right)^{-1} \sum_{m=0}^{\infty} \boldsymbol{P}_{m+1}(T) \Delta u^{(m)}(t) .
$$

The final step in our derivation of the small-signal model uses $x$ to reconstruct the amplifier output. From (1), it follows that the Fourier transform of the full output $g(t)$ is

$$
\hat{g}(\omega)=\int_{-\infty}^{\infty} \mathrm{e}^{-\mathrm{i} \omega t} g(t) \mathrm{d} t=\frac{2}{\mathrm{i} \omega} \sum_{n=-\infty}^{\infty}\left(\mathrm{e}^{-\mathrm{i} \omega n T}-\mathrm{e}^{-\mathrm{i} \omega A_{n}}\right)
$$

where the last inequality holds for $\omega \neq 0$. By considering the difference between the Fourier transform of the output with and without perturbation, we find that the perturbation to the output has Fourier transform (for $\omega \neq 0$ )

$$
\Delta \hat{g}(\omega)=\frac{2}{\mathrm{i} \omega} \sum_{n=-\infty}^{\infty}\left(\mathrm{e}^{-\mathrm{i} \omega \bar{A}_{n}}-\mathrm{e}^{-\mathrm{i} \omega A_{n}}\right) .
$$


Linearisation in small perturbations then gives

$$
\mathrm{e}^{-\mathrm{i} \omega A_{n}}=\mathrm{e}^{-\mathrm{i} \omega \bar{A}_{n}} \mathrm{e}^{-\mathrm{i} \omega \Delta a_{n} T} \sim \mathrm{e}^{-\mathrm{i} \omega \bar{A}_{n}}\left(1-\mathrm{i} \omega \Delta a_{n} T\right) .
$$

(In the time domain, this linearisation is tantamount to replacing the narrow rectangular pulses in $\Delta g(t)$ by Dirac $\delta$-functions.) Thus (34) becomes

$$
\Delta \hat{g}(\omega)=2 T \sum_{n=-\infty}^{\infty} \mathrm{e}^{-\mathrm{i} \omega \bar{A}_{n}} x\left(\bar{A}_{n}\right)=2 \sum_{n=-\infty}^{\infty} \mathrm{e}^{-2 \pi n \mathrm{i} a} \hat{x}(\omega-2 \pi n / T),
$$

where the second equality follows from Poisson resummation [24].

There is no particular restriction on the bandwidth of the input perturbation signal for (35) to be valid. However, further mathematical progress is substantially eased if we make the physically reasonable assumption that the input perturbation $\Delta u(t)$ contains only audio frequencies, so that $\Delta u(t)$ (and hence also $x(t)$ ) is band-limited, with $\Delta \hat{u}(\omega)=\hat{x}(\omega)=0$ for $|\omega| \geq \pi / T$; then, from (33) and (35),

$$
\Delta \hat{g}(\omega)=\kappa \gamma^{T}\left(\mathrm{e}^{\mathrm{i} \omega T} I_{5}-\mathcal{N}\right)^{-1} \sum_{m=0}^{\infty} \boldsymbol{P}_{m+1}(T)(\mathrm{i} \omega)^{m} \Delta \hat{u}(\omega)
$$

for $|\omega|<\pi / T$. To simplify the sum in this expression, we let

$$
\boldsymbol{\sigma}(T ; \mathrm{i} \omega)=\sum_{m=0}^{\infty} \boldsymbol{P}_{m+1}(T)(\mathrm{i} \omega)^{m}
$$

then note that in consequence

$$
\frac{\mathrm{d} \boldsymbol{\sigma}(T ; \mathrm{i} \omega)}{\mathrm{d} T}-\mathrm{i} \omega \boldsymbol{\sigma}(T ; \mathrm{i} \omega)=\boldsymbol{P}_{0}(T), \quad \boldsymbol{\sigma}(0 ; \mathrm{i} \omega)=\mathbf{0} .
$$

Solving this ODE, we thus have

$$
\boldsymbol{\sigma}(T ; \mathrm{i} \omega)=\sigma_{1} \boldsymbol{e}_{1}+\sigma_{2} \boldsymbol{e}_{2}+\sigma_{3} \boldsymbol{e}_{3}
$$

where

$$
\begin{aligned}
\sigma_{1} & =\frac{\mathrm{e}^{\mathrm{i} \omega T}-1}{\mathrm{i} \omega}, \\
\sigma_{2} & =\frac{\mathrm{i} \omega \sin \omega_{1} T-\mathrm{i} \omega_{1} \sin \omega T+\omega_{1}\left(\cos \omega_{1} T-\cos \omega T\right)}{\omega_{1}\left(\omega^{2}-\omega_{1}^{2}\right)} \\
\sigma_{3} & =\frac{\omega \sin \omega_{1} T-\omega_{1} \sin \omega T}{\omega \omega_{1}\left(\omega^{2}-\omega_{1}^{2}\right)}+\mathrm{i} \frac{\omega_{1}^{2} \cos \omega T-\omega^{2} \cos \omega_{1} T+\omega^{2}-\omega_{1}^{2}}{\omega \omega_{1}^{2}\left(\omega^{2}-\omega_{1}^{2}\right)} .
\end{aligned}
$$

This, finally, yields the (input-output) transfer function, from $\Delta \hat{u}(\omega)$ to $\Delta \hat{g}(\omega)$, which is

$$
\kappa \gamma^{T}\left(\mathrm{e}^{\mathrm{i} \omega T} I_{5}-\mathcal{N}\right)^{-1} \boldsymbol{\sigma}(T ; \mathrm{i} \omega)
$$


for "audio frequencies" (those less than $\pi / T$ in magnitude).

Without RC, this transfer function depends on $u_{0}$, through the value of $\kappa$ (both explicitly in (36) and implicitly through $\mathcal{N}$ ) and so the small-signal model predicts an inherently nonlinear response for the amplifier. This is undesirable, since such nonlinearity leads to unwanted total harmonic distortion (THD) and intermodulation distortion (IMD) [12, 25].

With RC, the transfer function is independent of $u_{0}$, so we expect it to provide an accurate characterisation of the input-output relation even for inputs that are not small perturbations to some constant input. This is a striking result, because it predicts an essentially linear behaviour for the amplifier. More specifically, for an audio input $u(t)$, the small-signal model predicts an output with audio-frequency Fourier components given by

$$
\hat{g}_{a}(\omega)=\kappa \gamma^{T}\left(\mathrm{e}^{\mathrm{i} \omega T} I_{5}-\mathcal{N}\right)^{-1} \boldsymbol{\sigma}(T ; \mathrm{i} \omega) \hat{u}(\omega)
$$

In fact, as we shall demonstrate in the next section, the full audio output is not quite linearly related

to the input: harmonics are generated, but from terms neglected in the small-signal linearisation

(cf. [10]). An example of such a term is $\left(\left(u^{\prime}\right)^{2}\right)^{\prime}$, which involves a product of input derivatives; the contribution of such terms is, however, small [10].

We next turn to a full calculation of the output that is not constrained by the linearisation inherent in the small-signal model.

\section{Fully nonlinear model}

Our final calculation gives the nonlinear audio output in response to a general audio input. This calculation tracks the slowly changing operating point of the amplifier in response to its input, in sufficient detail to allow us to find the principal contributions to the output distortion. Of necessity, it avoids the traditional quasi-steady engineering approximation, that the input to the amplifier is assumed constant over any switching cycle. We follow the structure of the calculation described in [8, 9, 10, 11, 12], although here the details are considerably more algebraically involved than in any of those previous cases. We emphasise that our approach may be readily adapted to other pulse-modulated feedback systems [4] with slowly varying input parameters.

We apply a perturbation method based on the small parameter

$$
\epsilon=\omega T \ll 1,
$$


where $\omega$ is a typical audio frequency. We introduce a correspondingly scaled time

$$
\tau=\omega t=\epsilon t / T \text {. }
$$

Thus variations to the audio input occur on a time scale $\tau=O(1)$, while the switching time scale has $\tau=O(\epsilon)$. We introduce

$$
\mathcal{U}(\tau)=u(t)
$$

so that $u^{(m)}(t)=(\epsilon / T)^{m} \mathcal{U}^{(m)}(\tau)$. Our interest is in determining how solutions to the system (11), (12) track the slow parametric variation afforded by the input audio signal.

We first determine the way in which the switching times depend on the audio input, then calculate the corresponding audio output. To this end, we introduce functions $a$ and $\mathcal{X}$ such that

$$
a(\epsilon n)=a_{n}, \quad \mathcal{X}(\epsilon n)=\boldsymbol{x}\left(A_{n}\right)=\boldsymbol{x}((n+a(\epsilon n)) T) .
$$

Writing the difference equation (11) and switching condition (12) in this notation, we find that each equation involves $a(\epsilon n)$ or $a(\epsilon(n+1))$. Clearly these equations are expected to hold only for integer values of $n$. However, as a mathematical device to enable a solution to be obtained, we seek to impose each equation for all real values of $n$ (since if we are able to do so then the equations certainly hold when restricted to integer $n$ ). Thus we set $\tau=\epsilon n$ and solve for all $\tau$ the following:

$$
\mathcal{X}(\tau+\epsilon)=\mathrm{e}^{N d_{\tau}} \mathcal{X}(\tau)+\boldsymbol{\Theta}(\tau), \quad \boldsymbol{\gamma}^{T} \mathcal{X}(\tau)=-1+2 a(\tau)
$$

where

$$
\begin{aligned}
\boldsymbol{\Theta}(\tau)= & \sum_{m=0}^{\infty} \frac{\epsilon^{m}}{T^{m}} \boldsymbol{P}_{m+1}\left(d_{\tau}\right) \mathcal{U}^{(m)}(\tau+\epsilon a(\tau))+\frac{2(1-k) \boldsymbol{Q}_{1}(a(\tau+\epsilon) T)}{L C} \\
& -\frac{(1+k-2 k a(\tau)) \boldsymbol{Q}_{1}\left(d_{\tau}\right)}{L C}+\frac{2 k}{T L C} \boldsymbol{Q}_{2}\left(d_{\tau}\right)
\end{aligned}
$$

and where

$$
d_{\tau}=(1+a(\tau+\epsilon)-a(\tau)) T
$$

The functions $a$ and $\mathcal{X}$ are then expanded in powers of $\epsilon$, and coefficients of successive powers of $\epsilon$ equated in (37).

Given the algebraic complexity of the perturbation problem, it is useful to reduce the problem from the six scalar equations represented in (37) to a single scalar equation, for $a(\tau)$ (cf. [16]). To do so, we introduce

$$
\mathcal{V}(\tau)=\mathrm{e}^{-a(\tau) N T} \mathcal{X}(\tau)
$$


Then the first of (37) becomes

$$
\mathcal{V}(\tau+\epsilon)=\mathrm{e}^{N T} \mathcal{V}(\tau)+\mathrm{e}^{-a(\tau+\epsilon) N T} \boldsymbol{\Theta}(\tau)
$$

where

$$
\zeta_{1}=\frac{1}{\epsilon \mathrm{D}} \sum_{n=0}^{\infty} \frac{B_{n}}{n !}(\epsilon \mathrm{D})^{n}, \quad \zeta(z)=\mathrm{e}^{-z T} \sum_{n=0}^{\infty} \frac{\beta_{n+1}\left(\mathrm{e}^{-z T}\right)}{(n+1) !}(\epsilon \mathrm{D})^{n},
$$

and the equation for $a(\tau)$ simplifies from (41) to

$$
\gamma^{T} R \mathrm{e}^{a(\tau) \Lambda T} \mathcal{D}\left\{\mathrm{e}^{-a(\tau+\epsilon) \Lambda T} R^{-1} \boldsymbol{\Theta}(\tau)\right\}=-1+2 a(\tau) .
$$

From the Fourier transform of (1), it may be deduced [8, 9, 10, 11] that the audio contribution to the output (i.e., the contribution involving frequencies less than $\pi / T$ ) is

$$
g_{a}(t)=-1+2 \sum_{n=0}^{\infty} \frac{(-\epsilon)^{n}}{(n+1) !} \frac{\mathrm{d}^{n} a^{n+1}(\tau)}{\mathrm{d} \tau^{n}}
$$


and so, in principle, the required calculation is now clear: we expand $a(\tau)$ in powers of $\epsilon$, as

$$
a(\tau)=a_{0}(\tau)+\epsilon a_{1}(\tau)+O\left(\epsilon^{2}\right)
$$

Writing $\boldsymbol{\Theta}(\tau)=\boldsymbol{\Theta}_{0}(\tau)+\epsilon \boldsymbol{\Theta}_{1}(\tau)+O\left(\epsilon^{2}\right)$, we find that

$$
\boldsymbol{\Theta}_{0}(\tau)=\boldsymbol{P}_{1}(T) \mathcal{U}(\tau)+\frac{2(1-k) \boldsymbol{Q}_{1}\left(a_{0}(\tau) T\right)}{L C}-\frac{\left(1+k-2 k a_{0}(\tau)\right) \boldsymbol{Q}_{1}(T)}{L C}+\frac{2 k}{T L C} \boldsymbol{Q}_{2}(T)
$$

and

$$
\begin{aligned}
\boldsymbol{\Theta}_{1}(\tau)= & \frac{1}{T} \boldsymbol{P}_{2}(T) \mathcal{U}^{\prime}(\tau)+\boldsymbol{P}_{1}(T) a_{0}(\tau) \mathcal{U}^{\prime}(\tau)+T \boldsymbol{P}_{0}(T) a_{0}^{\prime}(\tau) \mathcal{U}(\tau) \\
& +\frac{2\left(k \boldsymbol{Q}_{1}(T)+(1-k) T \boldsymbol{Q}_{0}\left(a_{0}(\tau) T\right)\right)\left(a_{1}(\tau)+a_{0}^{\prime}(\tau)\right)}{L C}-\frac{\left(1+k-2 k a_{0}(\tau)\right) T \boldsymbol{Q}_{0}(T) a_{0}^{\prime}(\tau)}{L C}
\end{aligned}
$$

In solving (42) for $a_{0}(\tau)$ and $a_{1}(\tau)$, we need the following expansions:

$$
\begin{aligned}
\mathrm{e}^{a(\tau) \Lambda T} & =\mathrm{e}^{a_{0}(\tau) \Lambda T}+\epsilon a_{1}(\tau) \mathrm{e}^{a_{0}(\tau) \Lambda T} \Lambda T+O\left(\epsilon^{2}\right), \\
\mathrm{e}^{-a(\tau+\epsilon) \Lambda T} & =\mathrm{e}^{-a_{0}(\tau) \Lambda T}-\epsilon\left(a_{1}(\tau)+a_{0}^{\prime}(\tau)\right) \mathrm{e}^{-a_{0}(\tau) \Lambda T} \Lambda T+O\left(\epsilon^{2}\right) .
\end{aligned}
$$

We also expand

$$
\left(\mathrm{e}^{\epsilon \mathrm{D}} I_{5}-\mathrm{e}^{\Lambda T}\right)^{-1}=\frac{1}{\epsilon \mathrm{D}} \Upsilon_{-1}+\Upsilon_{0}+O(\epsilon)
$$

where $\Upsilon_{-1}=\operatorname{diag}(1,0,0,0,0)$ and

$$
\Upsilon_{0}=\operatorname{diag}\left(-1 / 2,\left(1-\mathrm{e}^{\mathrm{i} \omega_{1} T}\right)^{-1},\left(1-\mathrm{e}^{-\mathrm{i} \omega_{1} T}\right)^{-1},\left(1-\mathrm{e}^{(-\mu+\mathrm{i} \Omega) T}\right)^{-1},\left(1-\mathrm{e}^{(-\mu-\mathrm{i} \Omega) T}\right)^{-1}\right) .
$$


The leading terms in (42) are those at $O\left(\epsilon^{-1}\right)$, which give

$$
\gamma^{T} \operatorname{Re}^{a_{0}(\tau) \Lambda T} \mathrm{D}^{-1}\left\{\Upsilon_{-1} \mathrm{e}^{-a_{0}(\tau) \Lambda T} R^{-1} \Theta_{0}(\tau)\right\}=0
$$

This equation may be considerably simplified by noting that $\Upsilon_{-1} \mathrm{e}^{-a_{0}(\tau) \Lambda T}=\Upsilon_{-1}$ and, further, that

$$
\Upsilon_{-1} \mathrm{e}^{-a_{0}(\tau) \Lambda T} R^{-1}=\mathcal{R}
$$
we may satisfy (47) by imposing the condition

$$
\boldsymbol{v}_{1} \boldsymbol{\Theta}_{0}(\tau)=0
$$

It is readily established that

$$
\boldsymbol{v}_{1} \boldsymbol{P}_{n}(t)=-\frac{t^{n}}{n ! L C}, \quad \boldsymbol{v}_{1} \boldsymbol{Q}_{n}(t)=-\frac{t^{n}}{n !},
$$

and hence, from (49),

$$
a_{0}(\tau)=\frac{1}{2}(1+\mathcal{U}(\tau))
$$

which is the analogue of the duty-cycle condition (15).

The next terms to consider in (42) are those at $O(1)$. After benefiting from the considerable simplification that follows from using (48) and imposing (49), we find

$$
\gamma^{T} \operatorname{Re}^{a_{0}(\tau) \Lambda T}\left(\Upsilon_{0} \mathrm{e}^{-a_{0}(\tau) \Lambda T} R^{-1} \Theta_{0}(\tau)+\mathrm{D}^{-1} \mathcal{R} \Theta_{1}(\tau)\right)=-1+2 a_{0}(\tau)
$$

Then, since $\exp \left( \pm a_{0}(\tau) \Lambda T\right)$ and $\Upsilon_{0}$ are all diagonal matrices, we see that

$$
\mathrm{e}^{a_{0}(\tau) \Lambda T} \Upsilon_{0} \mathrm{e}^{-a_{0}(\tau) \Lambda T}=\Upsilon_{0}
$$

furthermore, $\mathrm{e}^{a_{0}(\tau) \Lambda T} \mathcal{R}=\mathcal{R}$. Thus, by making use of these results and (48), we have

$$
\gamma^{T} R\left(\Upsilon_{0} R^{-1} \Theta_{0}(\tau)+D^{-1} \mathcal{R} \Theta_{1}(\tau)\right)=-1+2 a_{0}(\tau)
$$

which we may solve by taking

$$
\gamma^{T} R \mathcal{R} \Theta_{1}(\tau)=\mathcal{U}^{\prime}(\tau)-\gamma^{T} R \Upsilon_{0} R^{-1} \Theta_{0}^{\prime}(\tau)
$$

where we have used (50) to eliminate $a_{0}(\tau)$. 
Now $\mathcal{R} \Theta_{1}(\tau)=\left(\theta_{1}, 0,0,0,0\right)^{T}$, where

$$
\theta_{1}=\boldsymbol{v}_{1} \boldsymbol{\Theta}_{1}(\tau)=\frac{T}{L C}\left(g_{1}-\frac{1}{2}(1-k) \mathcal{U}(\tau) \mathcal{U}^{\prime}(\tau)\right),
$$

where $g_{1}$ is defined in (46). Furthermore, elementary matrix algebra gives

$$
\boldsymbol{\gamma}^{T} R \mathcal{R} \Theta_{1}(\tau)=\theta_{1} \boldsymbol{\gamma}^{T} \boldsymbol{w}_{1}=-\frac{L C}{\omega_{1}^{2}}\left(c_{1} \omega_{1}^{2}+c_{3}\right) \theta_{1}
$$

The right-hand side of (51) may be expressed more concretely by noting that

$$
\boldsymbol{\Theta}_{0}^{\prime}(\tau)=\boldsymbol{P}_{1}(T) \mathcal{U}^{\prime}(\tau)+\frac{(1-k) T}{L C} \boldsymbol{Q}_{0}\left(a_{0}(\tau) T\right) \mathcal{U}^{\prime}(\tau)+\frac{k}{L C} \boldsymbol{Q}_{1}(T) \mathcal{U}^{\prime}(\tau) .
$$

If we now define

$$
p_{n}(t)=\gamma^{T} R \Upsilon_{0} R^{-1} \boldsymbol{P}_{n}(t), \quad q_{n}(t)=\gamma^{T} R \Upsilon_{0} R^{-1} \boldsymbol{Q}_{n}(t)
$$

then $g_{1}$ is given by

$$
g_{1}=\frac{(1-k) \mathcal{U}(\tau) \mathcal{U}^{\prime}(\tau)}{2}-\frac{\omega_{1}^{2}(1-\psi(\tau)) \mathcal{U}^{\prime}(\tau)}{\left(c_{1} \omega_{1}^{2}+c_{3}\right) T}
$$

where

$$
\psi(\tau)=p_{1}(T)+\frac{(1-k) T}{L C} q_{0}\left(\frac{1}{2}(1+\mathcal{U}(\tau)) T\right)+\frac{k}{L C} q_{1}(T)
$$

This expression for $g_{1}$ enables us to determine the most significant components of the audio distortion.

We note that without $\mathrm{RC}$ (i.e., for $k=0$ ) the expression for $g_{1}$ is nonlinear in $\mathcal{U}$, and hence the output contains harmonic distortion at $O(\epsilon)$. We also see that with $\mathrm{RC}(k=1) g_{1}$ becomes the much simpler expression

$$
g_{1}=-\frac{\omega_{1}^{2}\left(1-p_{1}(T)-q_{1}(T) /(L C)\right)}{\left(c_{1} \omega_{1}^{2}+c_{3}\right) T} \mathcal{U}^{\prime}(\tau),
$$

which involves only terms that are linear in $\mathcal{U}$ (the first nonlinear terms, involving quantities such as $\left(\left(\mathcal{U}^{\prime}\right)^{2}\right)^{\prime}$, which involve three derivatives, will arise first at $O\left(\epsilon^{3}\right)$ in the output, cf. [10]).

Thus we have determined explicitly (at least, to $O(\epsilon)$ ) the way in which the nonlinear behaviour of the amplifier tracks the slowly varying audio input, and in particular the resulting low-frequency components of the output. While the leading-order tracking result, from (45) and (50), that $g_{a} \sim \mathcal{U}$, is well known and is easily understandable from the duty-cycle balance in (15), a comprehensive calculation of the type above is necessary to obtain a complete perturbative calculation of corrections. 


\begin{tabular}{ll}
\hline$R=8 \Omega$ & $c_{1}=1.3318 \times 10^{5} / \mathrm{s}$ \\
\hline$C=0.5169 \mu \mathrm{F}$ & $c_{2}=1.3763 \times 10^{10} / \mathrm{s}^{2}$ \\
\hline$L=10 \mu \mathrm{H}$ & $c_{3}=-1.0747 \times 10^{14} / \mathrm{s}^{3}$ \\
\hline$T=1 / 384000 \mathrm{~s}$ & $\omega_{1}=1.3195 \times 10^{5} \mathrm{rad} / \mathrm{s}$
\end{tabular}

Table 1: Parameter values used in simulations, unless otherwise specified.

\begin{tabular}{lll}
\hline Frequency $(\mathrm{kHz})$ & Analytical & Numerical \\
\hline 2 & $5.247 \times 10^{-5}$ & $5.258 \times 10^{-5}$ \\
\hline 3 & $2.23 \times 10^{-6}$ & $1.52 \times 10^{-6}$ \\
\hline 4 & $1.25 \times 10^{-5}$ & $1.38 \times 10^{-5}$ \\
\hline
\end{tabular}

Table 2: Absolute value of the Fourier components at various harmonics of the input $1 \mathrm{kHz}$ sine wave: analytical results from (45) and (52), and numerical results from simulation.

Our results confirm the conclusions of the small-signal model that RC (almost) completely linearises the output.

Although the perturbation calculation described in this section can, in principle, be taken to higher order in $\epsilon$, in practice the algebra required for this fifth-order system rapidly becomes unmanageable, even using computer algebra. Fortunately, the dominant contributions to the distortion seem to be captured by the terms to $O(\epsilon)$, for reasonable parameter values.

\section{Results}

For our first set of simulations, we take the parameter values in Table 1, These give stable steady-state operation, according to the criteria in Section 4. We carry out simulation of the amplifier in Matlab Simulink and compare results with the small-signal transfer function in (36) and with analytical predictions of the audio output from (45) and (52).

In the absence of $\mathrm{RC}(k=0)$, we examine a sine wave input

$$
u(t)=u_{*} \sin (2 \pi f t)
$$




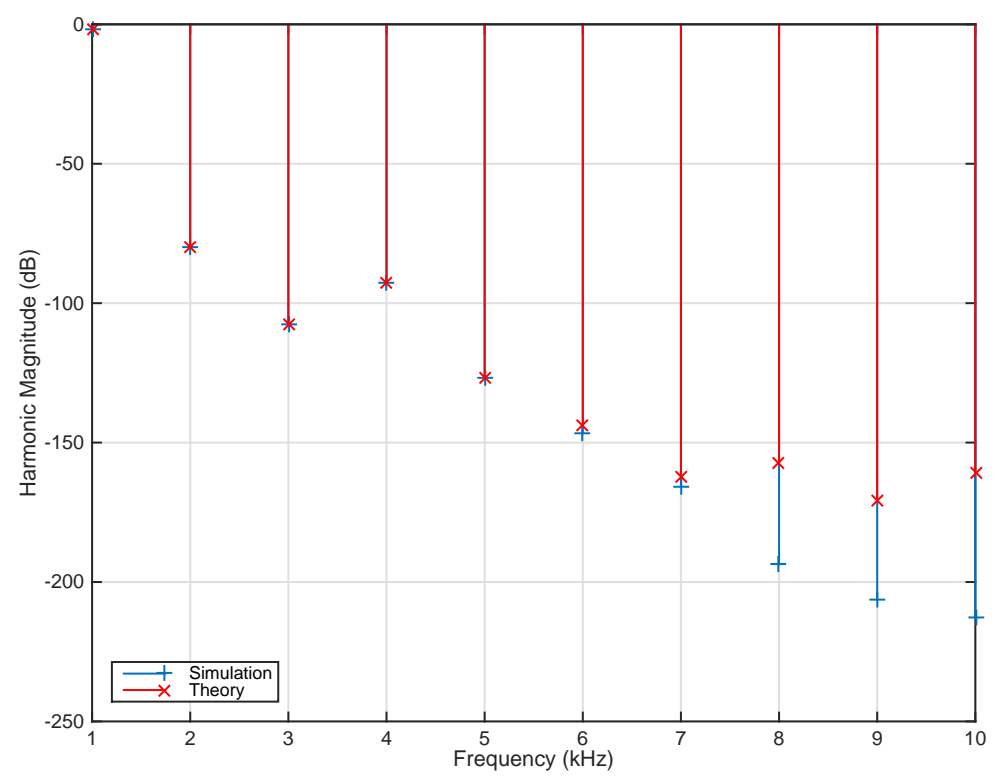

Figure 5: Spectrum of the PWM pulse train $p(t)$ without ripple compensation: analytical results from (45) and (52), and numerical results from simulation.

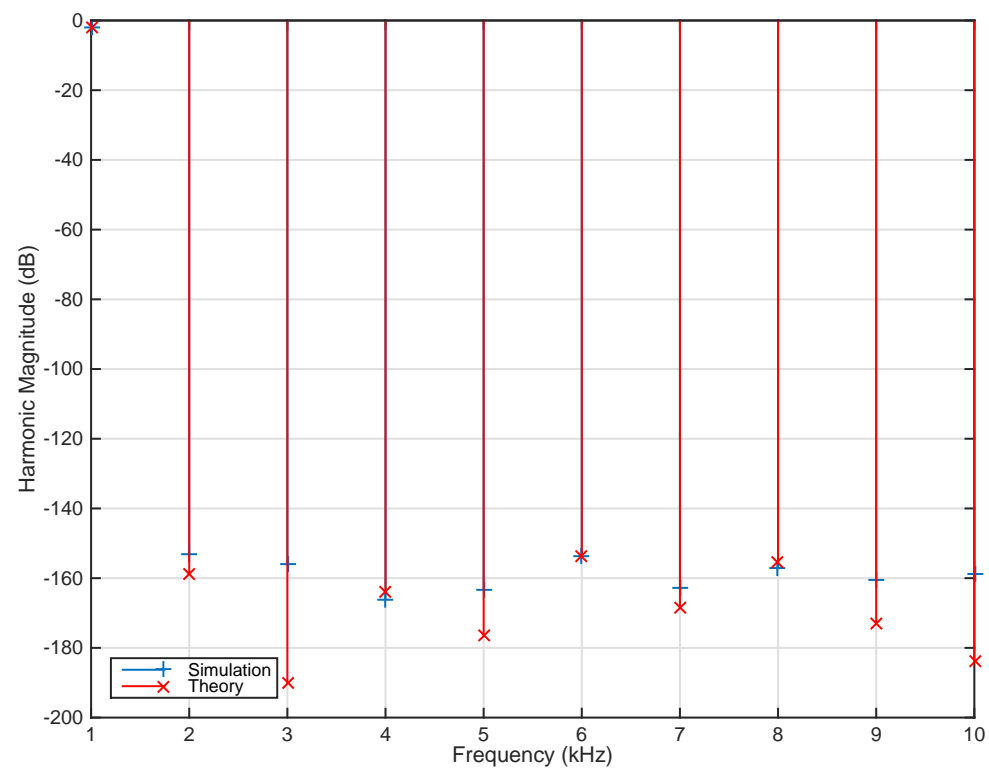

Figure 6: Spectrum of the PWM pulse train $p(t)$ with ripple compensation. 
with $u_{*}=0.8$ and $f=1 \mathrm{kHz}$. For the analytical result in (45), we keep terms at $O(1)$ and $O(\epsilon)$; the output Fourier component at the fundamental frequency is then predicted to be $-0.01356-0.4 \mathrm{i}$, while simulation gives $-0.0166-0.3988$. The absolute values of the Fourier components for the

second, third and fourth harmonics are given in Table 2, further results are summarised in Figure 5 , Given the small amplitude of harmonics and the small number of terms kept in the perturbation analysis, these results represent very good agreement between theoretical and numerical results. We note that in a practical implementation any harmonic below about $-140 \mathrm{~dB}$ will disappear beneath the noise floor, and will not be observable in measurement.

In the presence of RC, we may compare simulation results both with the perturbation calculation above, and also with the predictions of the small-signal model. Taking terms at $O(1)$ and $O(\epsilon)$, (45) predicts that the output contains only the fundamental. Its prediction of the amplitude of the output fundamental agrees exactly with predictions of the small-signal model, when the latter is appropriately truncated. When $u(t)$ is as in (53), with $u_{*}=0.8$ and $f=1 \mathrm{kHz}$, the output Fourier component at the fundamental frequency is analytically $-0.0135-0.3987 \mathrm{i}$, and from simulation -0.0166 - 0.3988i. Further results are summarised in Figure 6; we note that all harmonics beyond the fundamental are beneath the practical noise floor of $-140 \mathrm{~dB}$. With $f$ instead $2 \mathrm{kHz}$, the corresponding results are $-0.0263-0.3949 \mathrm{i}$ and $-0.0327-0.3952 \mathrm{i}$. At lower amplitude, with $u_{*}=0.5$ (and $f=1 \mathrm{kHz}$ ), the results are $-0.0084-0.2492 \mathrm{i}$ and $-0.0104-0.2492 \mathrm{i}$. Again the agreement between theoretical prediction and simulation is very good. The largest harmonic in the output is measured to be less than $10^{-5}$, which confirms the effectiveness of RC in eliminating higher harmonics from the output.

\subsection{Effects of instability}

The bifurcation structure of a negative-feedback pulse-modulated system such as described in this paper can be extremely intricate [5, 6] in response to a sinusoidal reference. However, the practical mode of operation for the present device aims to avoid instability; thus it is sufficient to use an approximation to the stability boundary, as we now describe. From either (26) or (28) we may determine whether the steady-state operating point in response to a constant input $u_{0}$ is stable or unstable. We find (either with or without RC) that the stability boundary depends only very weakly on the value of $u_{0}$. Correspondingly, we find that the steady-state stability threshold gives a very good indication of the stability of operation in response to an audio sine-wave input 


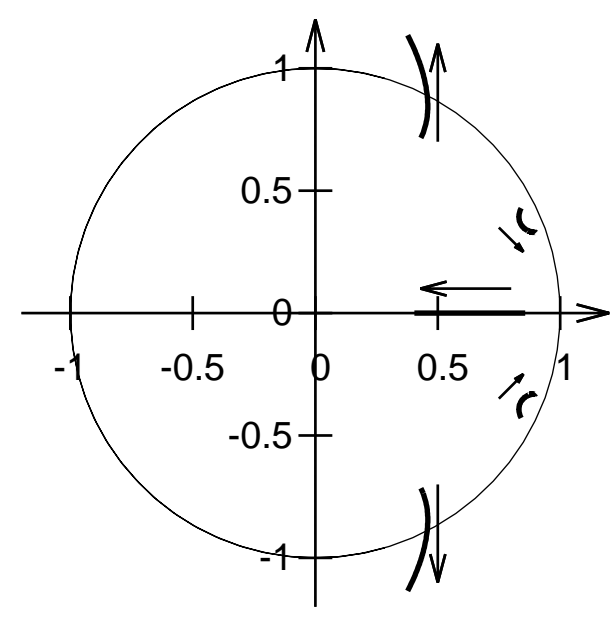

Figure 7: Path of the eigenvalues of $\mathcal{M}$ in the complex plane as $c_{1}$ is varied from $10^{5} / \mathrm{s}$ to $4.5 \times 10^{5} / \mathrm{s}$, all other parameter values being as in Table 1 and with $k=0$. Arrows indicate the direction for increasing values of $c_{1}$.

(i.e., one that varies slowly compared with the time scale of the switching). The behaviour of the amplifier is markedly different in the "stable" and "unstable" cases, and in practice the threshold between the cases is quite sharp.

For expository purposes, we use $c_{1}$ as our bifurcation parameter, holding all other parameters fixed at their values as in Table 1. The paths of the eigenvalues of the matrix $\mathcal{M}$ are shown in Figure 7 as $c_{1}$ is varied from $10^{5} / \mathrm{s}$ to $4.5 \times 10^{5} / \mathrm{s}$, for a constant input $u_{0}=0$, with $k=0$. In fact the eigenvalues vary little with the choice of $u_{0}$ or $k$. We find that the steady-state operating point is stable for $c_{1}<c_{1 c}$, where $c_{1 c}$ varies between $2.206 \times 10^{5} / \mathrm{s}$ and $2.208 \times 10^{5} / \mathrm{s}$ as $u_{0}$ varies in the interval $[-1,1]$. For practical purposes, it is thus a reasonable approximation to consider that there is a single point at which the bifurcation from stability to instability takes place (although a more detailed analysis would undoubtedly reveal a rich, finer-grained bifurcation structure [5, 6]). Instability of the steady-state operating point arises through a pair of complex conjugate eigenvalues of $\mathcal{M}$ leaving the unit circle, as in Figure 7 . Beyond the bifurcation point, there are corresponding oscillations in the duty cycle, which grow until $a_{n}$ reaches 0 or 1 , at which point the duty cycle saturates, and we observe one or more switching periods in which no switching in fact takes place (one or more pulses are "skipped" [3, 11, 27] ). This saturation of the duty cycle tends to occur most readily when $|u(t)|$ is greatest. The oscillations in $a_{n}$ and its saturation at 0 or 




Figure 8: Line $\mathrm{T}$ shows $\log _{10}$ THD as a function of $c_{1}$, all other parameters being as in Table 1. Lines 2, 3, 4, respectively, give $\log _{10}$ of the amplitudes of the second, third and fourth harmonics in the output. The input is $u(t)=0.8 \sin (2 \pi f t)$, with $f=1 \mathrm{kHz}$.

1 lead to a sudden calamitous jump in the amplitude of harmonics in the output, and consequent sudden steep rise in the total harmonic distortion (THD); see Figure 8 . The THD of a signal may be defined as follows:

$$
\text { for } f(t)=\sum_{-\infty}^{\infty} f_{n} \mathrm{e}^{n \mathrm{i} \omega t}, \quad \mathrm{THD}=\frac{\sqrt{\left|f_{2}\right|^{2}+\left|f_{3}\right|^{2}+\cdots}}{\left|f_{1}\right|}
$$

As is evident in Figure 8, there appears to be some uncertainty in our measurements of the harmonic amplitudes and the THD beyond the onset of instability, in contrast to our crisp results up to that point. The reason is that, prior to the onset of instability there are just two frequencies in the system, one associated with the audio sine wave and the other associated with the switching. Both frequencies are at our disposal; we choose these two frequencies to be commensurate and ensure that the time interval of simulation is an integer multiple of both the switching period $T$ and the sine-wave period $1 / f$. However, beyond the bifurcation point a third frequency is present in the simulations, relating to the oscillations in the duty cycle about the (now unstable) steadystate response. This third frequency arises dynamically in the system and is not a parameter at our disposal. Hence our simulations in general do not contain an integer number of periods 
of this oscillation. Consequently, there is spectral leakage [28] (absent before the instability) and our measurements of various harmonic components are correspondingly contaminated. However, beyond the bifurcation point the THD performance of the amplifier is so poor that a precise fidelity reproduction.

Of course our exploration of the high-dimensional parameter space of this amplifier is extremely limited: it is entirely possible that by choosing different parameter values and/or varying different parameters we might find a supercritical bifurcation leading to oscillations that saturate at small amplitude beyond the point of instability. In this case, any rise in THD is likely to be far less dramatic than that observed here.

\subsection{Comparison with open-loop design, and experimental results}

It is instructive to compare the foregoing results with those from an open-loop design. The latter involves just the carrier-wave generator and comparator from Figure 1, with the audio signal at the positive comparator input and the carrier wave at the negative input. The full output spectrum for the open-loop case is well known [29, 30, 31]: the distortion components in the audio spectrum are due entirely to sidebands of the PWM switching frequency, and are negligible, being smaller than the smallest numerical value that can be represented by Matlab's floating point 64bit format. However, despite its extremely low theoretical level of audio distortion, the open-loop design is less advantageous in practice than a closed-loop design, such as examined here, because the latter is considerably better at rejecting unwanted perturbations caused by disturbances to the power supply or other non-idealities of the physical device. Experimental results for a closed-loop class-D amplifier designed by making use of the proposed approach show (see Figure 13 of [32]) that the measured total harmonic distortion (THD) at $1 \mathrm{~W}$ is equal to $0.3 \%$ for the open-loop amplifier and decreases to $0.003 \%$ when the feedback loop is closed. Experimental results for a digitally controlled class-D amplifier also based on the proposed approach show [13] that a THD of $0.0028 \%$ is achieved at an output power of $1 \mathrm{~W}$. More detailed experimental results [33] show how the proposed approach results in a flat frequency response, a low output impedance across the audio band and very low inter-modulation distortion. 


\section{Acknowledgments}

We gratefully acknowledge the financial support of the South-African National Research Foundation (NRF). 


\section{References}

[1] M. Berkhout, L. Dooper, Class-D audio amplifiers in mobile applications, IEEE Trans. Circ. Syst. I 57 (2010) 992-1002.

[2] H. S. Black, Modulation Theory, Van Nostrand, New York, 1953.

[3] M. Di Bernardo, C. J. Budd, A. R. Champneys, P. Kowalczyk, Piecewise-Smooth Dynamical Systems: Theory and Applications, Applied Mathematical Sciences, vol. 163. Springer, London, 2008.

[4] A. Kh. Gelig, A. N. Churilov, Stability and Oscillations of Nonlinear Pulse-Modulated Systems, Birkhäuser, Boston, 1998.

[5] V. Avrutin, E. Mosekilde, Zh. T. Zhusubaliyev, L. Gardini, Onset of chaos in a single-phase power electronic inverter, Chaos 25 (2015) 043114.

[6] V. Avrutin, Zh. T. Zhusubaliyev, E. Mosekilde, Border collisions inside the stability domain of a fixed point, Physica D 321-322 (2016) 1-15.

[7] C. C. Fang, Sampled-Data Analysis and Control of DC-DC Switching Converters, PhD thesis, University of Maryland, College Park, 1997.

[8] S. M. Cox, B. H. Candy, Class-D audio amplifiers with negative feedback, SIAM J. Appl. Math. 66 (2005) 468-488.

[9] S. M. Cox, C. K. Lam, M. T. Tan, A second-order PWM-in/PWM-out class-D audio amplifier, IMA J. Appl. Math. 78 (2013) 159-180.

[10] S. M. Cox, H. du T. Mouton, Ripple compensation for a class-D amplifier, SIAM J. Appl. Math. 75 (2015) 1536-1552.

[11] S. M. Cox, M. T. Tan, J. Yu, A second-order class-D audio amplifier, SIAM J. Appl. Math. 71 (2011) 270-287.

[12] S. M. Cox, J. Yu, W. L. Goh, M. T. Tan, Intrinsic distortion of a fully differential BDmodulated Class-D amplifier with analog feedback, IEEE Trans. Circ. Syst. I 60 (2013) 63-73. 
[13] T. Mouton, B. Putzeys, Digital control of a PWM switching amplifier with global feedback, in: Audio Engineering Society Conference: 37th International Conference: Class D Audio Amplification, 2009.

[14] B. Putzeys, Simple, ultralow-distortion digital pulse width modulator, in: Audio Engineering Society Convention: 120th Convention: Paris, France, 2006.

[15] C. Neesgaard, L. Risbo, PWM amplifier control loops with minimum aliasing distortion. In 120th Audio Engineering Society Convention (2006) Audio Engineering Society.

[16] G. A. Papafotiou, N. I. Margaris, Calculation and stability investigation of periodic steady states of the voltage controlled buck DC-DC converter, IEEE Trans. Power Electr. 19 (2004) 959-970.

[17] M. A. Aizerman, F. R. Gantmakher, On the stability of periodic motions, J. Appl. Math. Mech. 22 (1958) 1065-1078.

[18] C. C. Fang, E. H. Abed, Robust feedback stabilization of limit cycles in PWM DC-DC converters, Nonlinear Dyn. 27 (2002) 295-309.

[19] C. C. Fang, E. H. Abed, Sampled-data modeling and analysis of closed-loop PWM DC-DC converters, in: Proceedings of the IEEE International Symposium on Circuits and Systems, ISCAS '99, Volume 5, 1999, pp. 110-115.

[20] C. C. Fang, E. H. Abed, Sampled-data modelling and analysis of the power stage of PWM DC-DC converters, Int. J. Electr. 88 (2001) 347-369.

[21] D. A. Harville, Matrix Algebra from a Statistician's Perspective, Springer, Berlin, 1997.

[22] G. Boole, A Treatise On The Calculus of Finite Differences (Second Edition), Macmillan, London, 1872.

[23] L. M. Milne-Thomson, The Calculus of Finite Differences, Macmillan, London, 1933.

[24] P. M. Morse, H. Feshbach, Methods of Theoretical Physics, Part I, McGraw-Hill, New York, 1953. 
[25] J. Yu, M. T. Tan, S. M. Cox, W. L. Goh, Time-domain analysis of intermodulation distortion of closed-loop class-D amplifiers, IEEE Trans. Power Electr. 27 (2012) 2453-2461.

[27] M. Di Bernardo, C. K. Tse, Chaos in power electronics: an overview, in: G. Chen, T. Ueta (Eds.) Chaos in Circuits and Systems, World Scientific Series on Nonlinear Science Series B: Volume 11, 2002, pp. 317-340.

[28] F. J. Harris, On the use of windows for harmonic analysis with the discrete Fourier transform,

[26] T. M. Apostol, On the Lerch zeta function, Pacific J. Math. 1 (1951) 161-167. Proc. IEEE 66 (1978) 51-83.

[29] D. G. Holmes, T. A. Lipo, Pulse Width Modulation for Power Converters: Principles and Practice. IEEE Press Series on Power Engineering, Vol. 18. John Wiley \& Sons, 2003.

[30] Z. Song, D. V. Sarwate, The frequency spectrum of pulse width modulated signals, Signal Proc. 83 (2003) 2227-2258.

[31] S. M. Cox, Voltage and current spectra for a single-phase voltage-source inverter, IMA J. Appl. Math. 74 (2009) 782-805.

[32] P. Kemp, T. Mouton, B. Putzeys, High-order analog control of a clocked class-D audio amplifier with global feedback using $z$-domain methods. In 131st Audio Engineering Society Convention (2011) Audio Engineering Society.

[33] P. J. Kemp, High-order analog control of a clocked class-D audio amplifier with global feedback using $z$-domain methods. Masters thesis, University of Stellenbosch, 2011. 